\title{
Degradation of light carrying orbital angular momentum by ballistic scattering
}

\author{
Shaun Viola $\odot,{ }^{1}$ Zhaozhong Chen, ${ }^{1}$ Alison M. Yao, ${ }^{2}$ Manousos Valyrakis $\odot,{ }^{1}$ Anthony E. Kelly, \\ David McKee, ${ }^{2}$ and Martin P. J. Lavery (1) ${ }^{1, *}$ \\ ${ }^{1}$ James Watt School of Engineering, University of Glasgow, Glasgow G12 8QQ, United Kingdom \\ ${ }^{2}$ Department of Physics, University of Strathclyde, Glasgow, United Kingdom
}

(Received 19 September 2019; revised 6 April 2020; accepted 15 May 2020; published 17 July 2020)

\begin{abstract}
Structured light can enhance the functionality of optical communication and sensing systems. Dense scattering environments such as those experienced in coastal water and foggy conditions result in degradation of structured optical fields. We present findings that characterize the degradation of the phase structure of ballistic scattered light carrying orbital angular momentum (OAM) propagated through a dense scattering medium over distances of up to $20 \mathrm{~m}$. We present a numerical channel modeling approach that can predict the scattering behavior at extended distances, which indicates that there is a strong mode-dependent variance in cross talk from the interaction of beams that carry OAM with randomly displaced scattering particles. These results present an effect that could allow the use of OAM modes to enhance particulate size sensors and could potentially lead to the development of novel tools for monitoring particles in underwater or free-space optical channels.
\end{abstract}

DOI: 10.1103/PhysRevResearch.2.033093

\section{INTRODUCTION}

The drive for high-bandwidth, secure communications links has led to space-division multiplexing (SDM) becoming a burgeoning area of study, where multiplexing-independent spatial channels can act to increase the capacity of communication links. SDM techniques implemented in systems employing both optical and radio-frequency carriers have recently received interest from the community for use within point-to-point communication links, particularly in longdistance fiber links [1-3]. In recent years the utilization of visible-light communications (VLC) has become an emerging technology with the potential to replace the currently widely implemented ultrasound underwater communication technology [4-6]. Such VLC schemes could potentially be enhanced through the addition of SDM [4,7]. Orbital angular momentum (OAM) multiplexing has become the SDM technique that many have chosen to explore [2,8-13]. Although demonstrations of OAM multiplexing in an underwater communications channel have been achieved in a controlled environment, it is important to present an understanding of the channel characteristics that would occur in a practical optical link. Localized heating, turbidity, salinity, and mechanical mixing, such as flowing water, all result in degradation of the optical field [14]. Developing a reliable and realistic channel model for scattering effects in water is critical for future implementations of SDM-enhanced submersed links.

*martin.lavery@glasgow.ac.uk

Published by the American Physical Society under the terms of the Creative Commons Attribution 4.0 International license. Further distribution of this work must maintain attribution to the author(s) and the published article's title, journal citation, and DOI.
Scattering from suspended particulates and optical aberrations from density variations are the main sources of channel degradation in any environment [13,15-19]. Suspended particulates further result in the attenuation of a propagating optical beam, and local deflection of light incident on these particles leads to multipath interference (Fig. 1). In coastal, river, and marine environments particulates have broad distributions of particle size that will collectively affect the propagation dynamics of an optical beam. The density of particles strongly influences water-attenuation measurements. Measurements of transmitted or scattered intensity over short path lengths are subsequently used with optical models to predict collective losses over longer propagation channels. Thermal variations in water due to current flow and mixing between stratified layers in the water column can further degrade an optical field in a manner similar to atmospheric turbulence. Exploring the validity of extending the small sample measurements $(<3 \mathrm{~m})$ to long distances $(>20 \mathrm{~m})$ is an important goal for confirming the deployment challenges of underwater SDM and could highlight possible methods for determining particulate content of water channels at long distances that could be beneficial for environmental monitoring. In this paper we outline a technique for modeling the propagation of spatially structured optical modes over underwater optical links. Optical beams carrying OAM, such as Laguerre Gaussian modes, are one of many complete mode sets that are widely considered for spatial multiplexing. OAM modes are chosen for this investigation as they have exhibited unique behavior in atmospheric environments that could be mirrored in scattering environments such as natural waters. The model is directly compared with an experimentally recorded result from a 3-m underwater link [Figs. 2(a) and 2(b)] and a 20-m link comprising multiple planes of scattering [Fig. 2(c)] that simulates the conditions of a coastal environment. The numerical model considers the effects arising from suspended particles in 


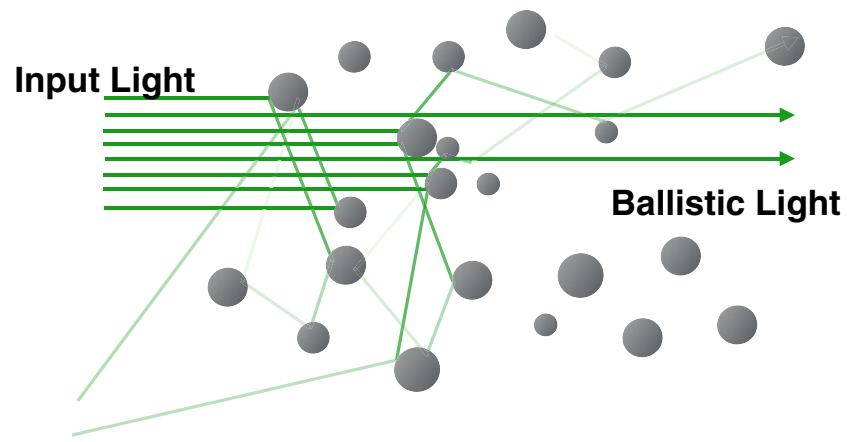

FIG. 1. Optical scattering from particulates is a concern with a submersed optical link. These particles move with the fluid flow and can result in aberrations, attenuation, and scattering for propagating optical fields.

thermally constant environments to explore purely the extension of the scattering behavior to longer distances. Our simulation indicates that distortion of OAM modes due to their interaction with particulates in turbid channels is sensitive to the particle size of randomly distributed particles in a turbid environment.

\section{INTERACTION WITH DISTRIBUTED PARTICLES}

Our underwater experimental link has an optical length of $3 \mathrm{~m}$ within a larger flow channel of $15 \mathrm{~m}$ in length. To replicate coastal conditions, a coarse uniform layer of gravel approximately $10 \mathrm{~cm}$ deep lines the base. As water flows through the channel, the gravel is agitated, leading to naturally buoyant silt particles being vigorously mixed and suspended throughout the water column. These small suspended particles scatter the light, reducing the overall power in the system, and increase the effective turbidity in the water. The channel is not initially heated, leading to a relatively small thermal variation within the water. To prepare the required optical modes a spatially filtered 532-nm laser source illuminates the surface of a spatial light modulator (SLM). An $\ell$-forked hologram is displayed on the optical surface of the SLM, generating the required OAM mode [20]. This optical hologram controls both the phase and intensity of the beam in the form $\Phi=A(r) \exp (i \ell \theta)$, where $A(r)$ is the radial intensity profile, $\ell$ is an unbounded integer quantifying the OAM carried by the beam, $r$ is the radial dimension, and $\theta$ is the azimuthal dimension. The optical mode is relayed using a periscopic mirror arrangement into a three-dimensional (3D) printed waterproof enclosure [Fig. 2(b)]. Transmission through an air-to-water boundary results in considerable optical aberration due to distortion at the water surface; hence, our optical modes are passed through a submersed optical window, reducing the effects from the fluctuating water surface.

The OAM spectrum after propagation over the underwater link was measured with the use of an OAM mode analyzer (MA). The MA uses two refractive optical elements that transform OAM states into transverse momentum states [21,22]. A lens can then separate the resultant transverse momentum states into specific lateral positions. A camera is placed at the focal plane of this lens, where 11 adjacent, equally sized regions were selected, each corresponding to a specific mode
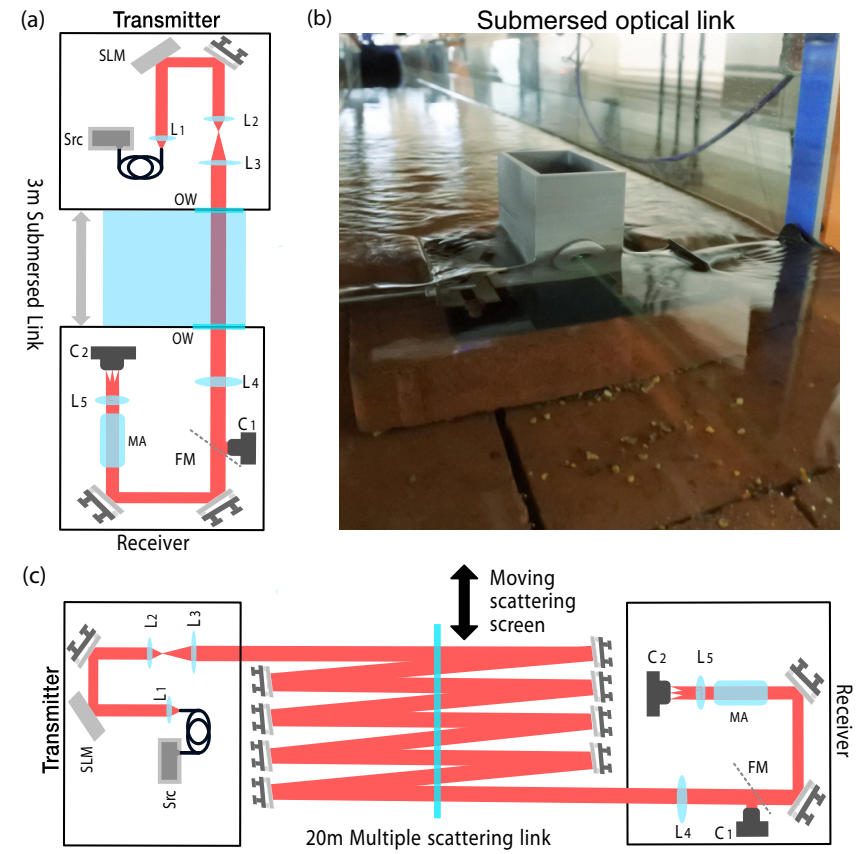

FIG. 2. (a) Schematic overview of an experimental setup to determine the optical aberrations that affect LG optical modes as they propagate through scattering silt particles. A laser source (Src) is collimated by a lens $\left(\mathrm{L}_{1}\right)$ and illuminates a spatial light modulator (SLM) to generate the desired optical profile. Two further lenses $\left(\mathrm{L}_{2}\right.$ and $\mathrm{L}_{3}$ ) pass through a toughened optical window $(\mathrm{OW})$ to enter the 3-m water channel. At the receiver a lens $\left(\mathrm{L}_{4}\right)$ is used to image the optical mode either onto a camera $\left(\mathrm{C}_{1}\right)$ or into a mode analyzer (MA) through the use of a flip mirror (FM). After the MA a final lens $\left(\mathrm{L}_{5}\right)$ focuses the light onto a camera to analyze the modal content of the propagating optical field $\left(\mathrm{C}_{2}\right)$. (b) To avoid distortions that arise from the top surface of the water, a $3 \mathrm{D}$ printed enclosure containing an elliptical mirror is used to act as a periscope to redirect the light into the submersed channel. (c) To consider the effect of variation in particle-size distributions at longer optical propagation lengths we constructed a 20-m-long optical link with a moving optical screen coated in randomly displaced particulates to replicate the suspended particles in a submersed channel.

index. The total power over all the pixels in each region was summed to give the power in that mode, allowing for the efficient measurement of the constituent OAM states simultaneously [23].

An equivalent optical setup was constructed to experimentally determine scattering effects at longer propagation lengths of up to $20 \mathrm{~m}$ [Fig. 2(c)]. Due to the constraints on the length of the flow channel and the challenges of precise alignment in an underwater channel this link was built on an optical table using a folded optical beam path. The optical scattering was achieved by multiple passes through a scattering screen coated in particles of an approximately known size. The rest of the optical system was matched to the underwater channel discussed above. Two particle sizes were investigated with average particle sizes of 11 and $50 \mu \mathrm{m}$, which are Maalox suspension and corn flour, respectively [24]. We note that as both are commercial products, some variance is expected in the particulate distribution. 

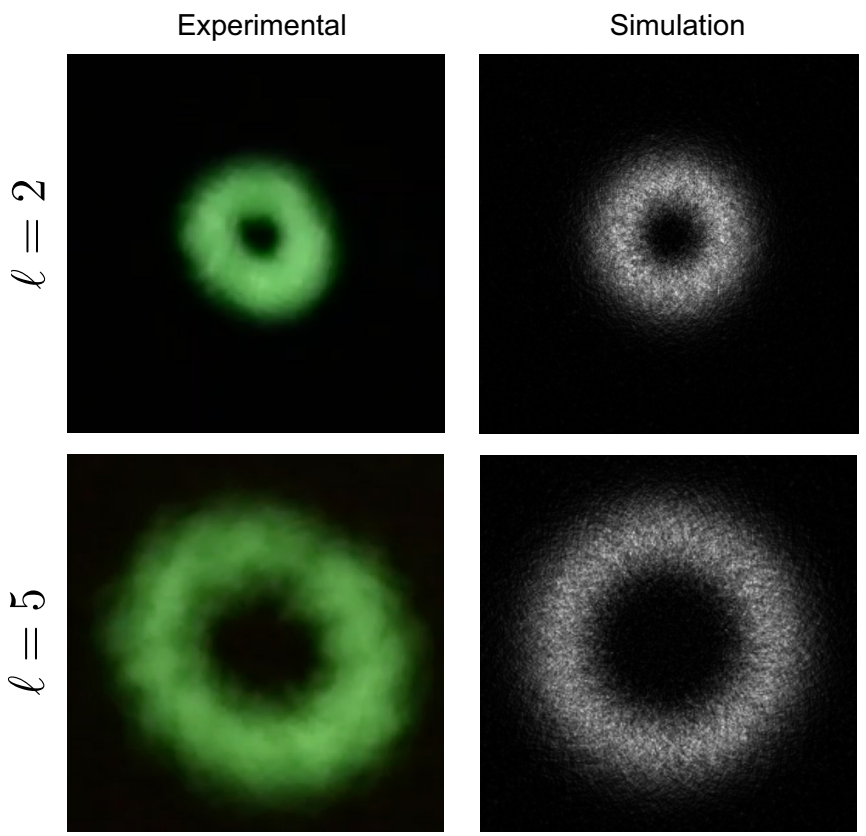

FIG. 3. The beam profiles were captured using a full-color CMOS camera (Nikon $1 \mathrm{~J}$ 4), indicated as $\mathrm{C}_{1}$ in Fig. 2. To assess the validity of our propagation model, we directly compare the mode profiles for $\ell=2$ and $\ell=5$, respectively.

Within any point-to-point to communications link, tip-tilt aberration is one of the largest concerns. Within oceanic conditions, the flow of the water potentially places added stress on the optical system and could perturb the optical beam, resulting in tip-tilt aberrations [4]. A mode range of $\ell=-5$ to $\ell=+5$ was chosen, and the power measured in each detection region was averaged over a 10 -s measurement window. Independent of turbulence, our mode sorter has a residual degree of cross talk between channels due to the diffraction limit and issues arising from the skew angle of the rays [22,25]. The channel cross talk for each of the 11 modes was measured to determine the experimental modal degradation arising from the turbid environment (Fig. 3).

\section{NUMERICAL MODELING OF CHANNELS}

To fully model the optical interaction with a channel comprising particles distributed evenly over a particular volume of water (or air), the channel is represented as a cascaded set of successive scattering screens with randomly displaced circular absorbing regions with radius $r_{p}$, corresponding to the size of simulated floating particles (see Fig. 4). Such an approach allows for the simulation of a large number of distributed particles $\left(>10^{5}\right.$ particles for sizes of $\left.11 \mu \mathrm{m}\right)$, which would be computationally challenging in ray-tracing approaches. For a given input optical beam we model the propagation between the cascaded scattering screens, separated by $10 \mathrm{~cm}$, computationally by plane-wave decomposition to calculate the spatial intensity and phase profiles of a propagating field. This split-step channel-simulation approach is repeated over a number of steps $N_{s}$ to simulate the accumulative effects of a long-distance scattering channel. To compute each scattering screen, we first determine the number of absorbing
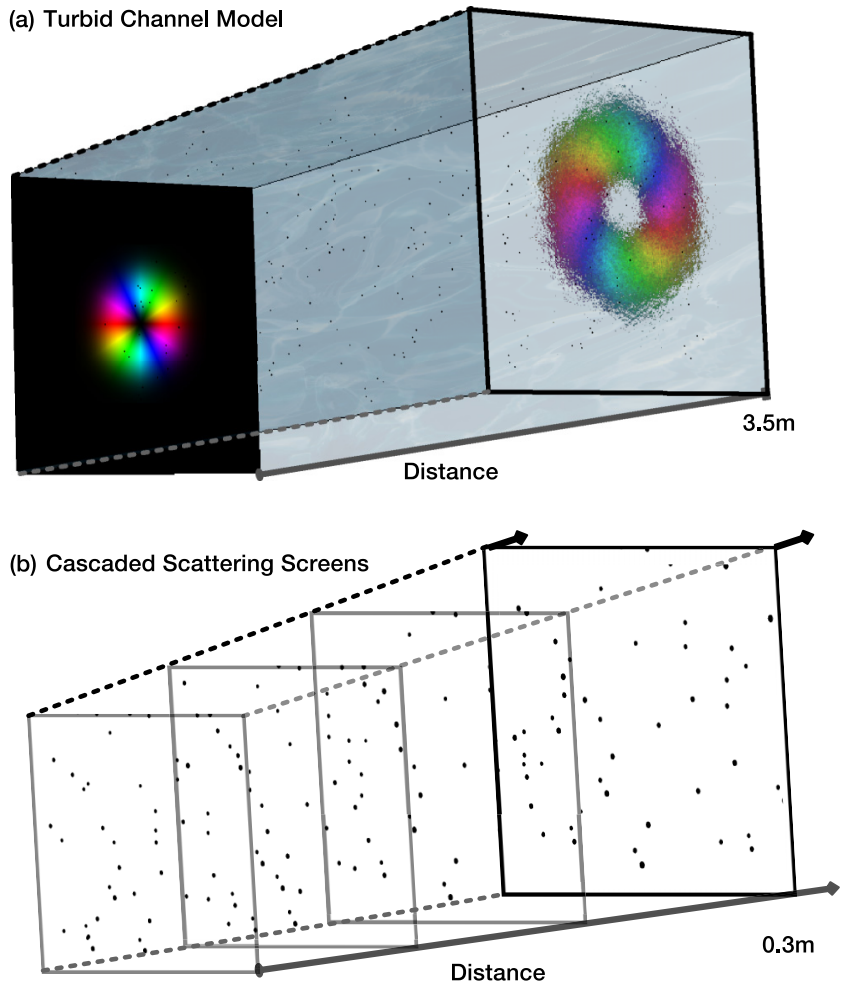

FIG. 4. (a) Laguerre-Gaussian (LG) modes are propagated through a series of cascaded phase screens comprising randomly distributed absorbing particles. (b) A subsection of the cascaded scattering screens, with $10 \mathrm{~cm}$ of propagation between each; many scattering screens are combined to simulate the scattering environment.

particles required in each scattering screen by considering the total channel loss $A_{T}$, where the expected loss for each scattering screen is the ratio $A_{s}=A_{T} / N_{s}$. By considering the area illuminated by the simulated input beam and the total particulate area coverage of absorbers that would result in a loss equivalent to $A_{s}$ the number of simulated particles in each screen is calculated as

$$
N_{p}=\frac{A_{T} w_{0}^{2}}{N_{s} r_{p}^{2}},
$$

where $w_{0}$ is the beam size of the propagating optical field. In each scattering screen, $N_{p}$ circles are randomly placed in a 2048 by 2048 array with a pixel pitch of $0.5 \mu \mathrm{m}$. Our random-number generator distributes the particles across the entire phase screen, with an even random distribution. At each propagation step an absorbing boundary of $5 \%$ of the total simulation area is added to prevent numerical reintroduction of wave-front components that leave the simulation area. In this way our propagation model neglects secondary bounces and considers only the ballistic photons that would be received by a detector.

We use as our pure OAM states the Laguerre-Gaussian (LG) laser modes already mentioned above. We compute our mode profiles in Cartesian coordinates here, as this is convenient for numerical simulations on a pixelated twodimensional (2D) array. LG modes are described by two 

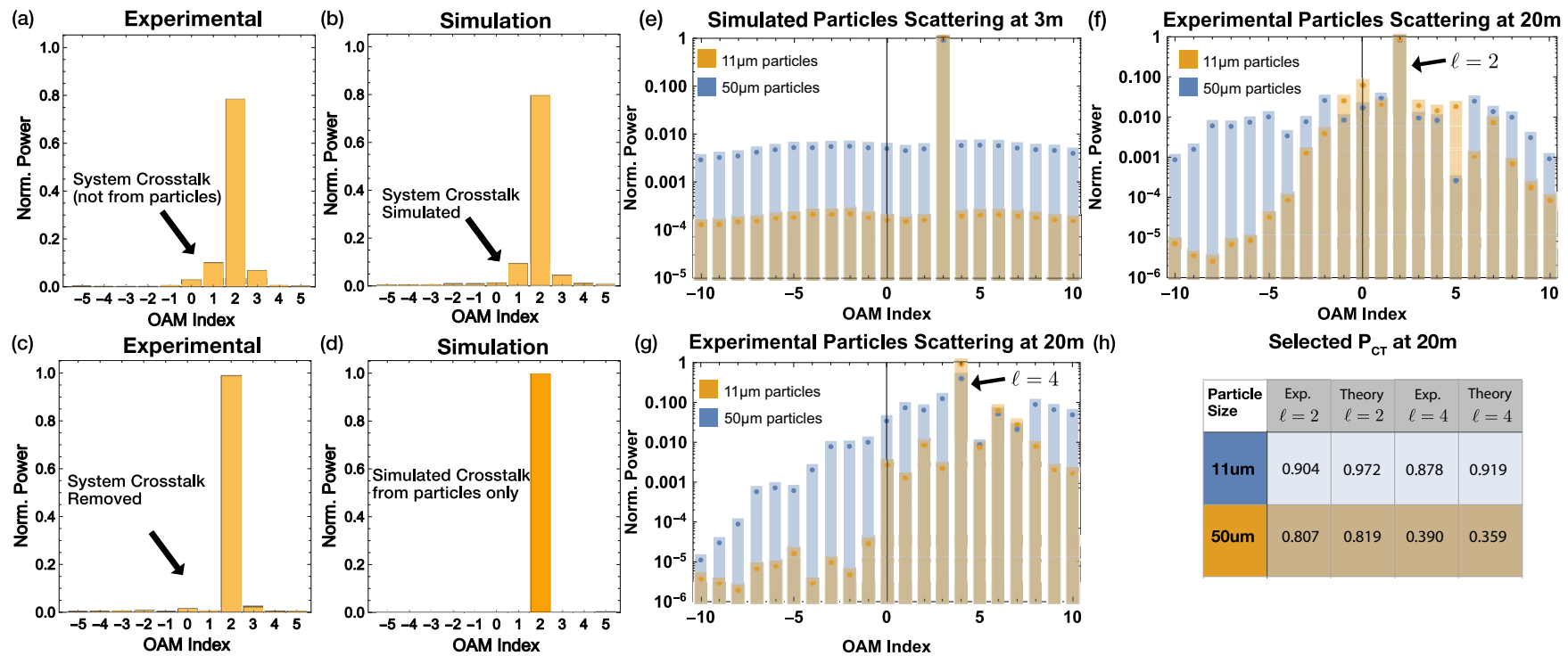

Selected $P_{C T}$ at $20 \mathrm{~m}$

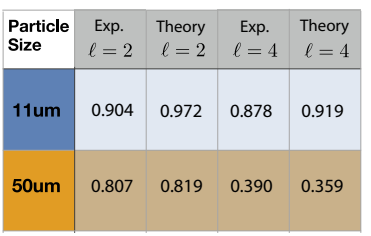

FIG. 5. (a) Experimentally measured channel cross talk for a mode with $\ell=2$ and $p=0$. Sidebands arise from systemic errors induced by the experimental system. (b) A numerical model for an equivalent channel and mode profile, showing very similar expected cross talk when simulating the full experimental system. (c) Systemic cross talk was removed to reveal no significant extra cross talk arising from the particles over the test channel, agreeing with other experimental studies [4,7] at this length scale on a linear scale. (d) Simulated cross talk assuming no systematic cross talk. (e) One can consider the expected measurements for an idealized mode analyzer with no inherent systematic cross talk. The scattering channel distributes a small amount of power into other modes. Changing the particulate size from $11 \mu \mathrm{m}$ (in yellow) to $50 \mu \mathrm{m}$ (blue) increases the cross talk for a propagation channel of $3 \mathrm{~m}$. Slight variations occur depending on the OAM mode propagation in the channel, which is more fully addressed in Fig. 6. Experimental measurements of cross talk for a propagation over a 20-m channel for (f) $\ell=2$ and (g) $\ell=4$. (h) Power distributed into other orders can be determined as the eigenvalues $P_{C T}$ of the power transfer matrix, Eq. (8), where the experimental results can be compared to theoretical results, showing good agreement.

indices, $\ell$ and $p$. In the waist plane of an LG mode the complex amplitude is given by

$$
\begin{aligned}
\Psi_{\ell, p}(x, y)= & C_{\ell, p}\left(\frac{\sqrt{2\left(x^{2}+y^{2}\right)}}{w_{0}}\right) L_{p}^{|\ell|}\left(\frac{2\left(x^{2}+y^{2}\right)}{w_{0}^{2}}\right) \\
& \times \exp \left(\frac{-\left(x^{2}+y^{2}\right)}{w_{0}^{2}}\right) \exp \left[i \ell \tan ^{-1}\left(\frac{y}{x}\right)\right],
\end{aligned}
$$

where $w_{0}$ is the waist size, $L_{p}^{|\ell|}(x)$ is the Laguerre polynomial for the mode indices $\ell$ and $p$, and $C_{\ell p}$ is the amplitude normalization term $[8,26]$, given by

$$
C_{\ell, p}=\frac{1}{w_{0}} \sqrt{\frac{2 p !}{\pi(p+|\ell|) !}} .
$$

Note that in evaluating the term $\exp \left[i \ell \tan ^{-1}\left(\frac{y}{x}\right)\right]$ numerically it is important to use an arctangent procedure that accounts for which quadrant the point $(x, y)$ is in.

The channel cross talk within the system was determined through two routes. Following the method outlined in [22] (Fig. 5), we first numerically simulated the optical transformation mode sorter to predict the systematic errors of the experimental system and confirm the validity of the propagation modeling. Second, we made use of the fact that the LG modes form a complete orthonormal basis set to write any beam cross section $\psi(x, y)$ as a superposition of LG modes:

$$
|\psi\rangle=\sum_{\ell=-\infty}^{\infty} \sum_{p=0}^{\infty} A_{p}^{\ell}|\ell p\rangle,
$$

where the contribution of each mode is given by

$$
A_{p}^{\ell}=\langle\ell p \mid \psi\rangle
$$

and the relative power in each mode is simply

$$
P_{\ell, p}=\left|A_{p}^{\ell}\right|^{2}=|\langle\ell p \mid \psi\rangle|^{2} .
$$

The power in all components with the same value of $\ell$ is given by

$$
P_{\ell}=\sum_{p=0}^{\infty}\left|A_{p}^{\ell}\right|^{2}=\sum_{p=0}^{\infty}|\langle\ell p \mid \psi\rangle|^{2} .
$$

The set of these powers $P_{\ell}$ is the OAM spectrum we expect to measure and hence represents the channel cross talk expected.

Initially, the scattering effects from the small randomly dispersed slit particles of $11 \mu \mathrm{m}$ are simulated to match the average size of the suspended particles in the experimental link. It can be seen in Figs. 5(a)-5(d) that experimental and numerical results are closely linked. Within the numerical system we can look at the cross-talk profile in greater detail as we are not limited by the inherent systematic errors in the experimental system. We consider the power distribution on a logarithmic scale to analyze the cross-talk variance with particulate size because for short-distance links one does 
(a)
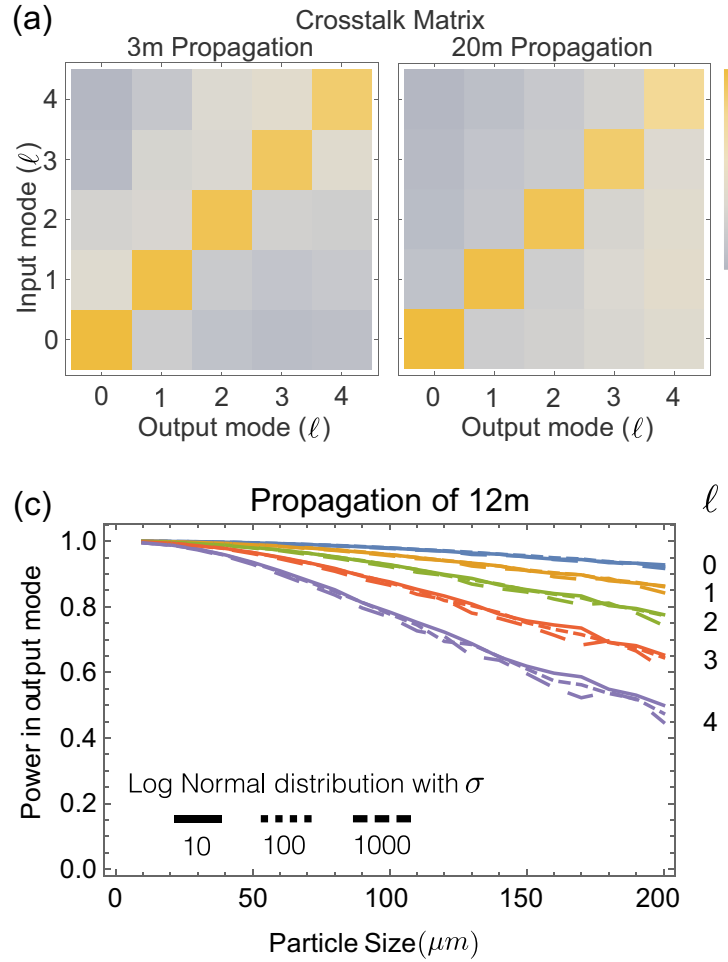
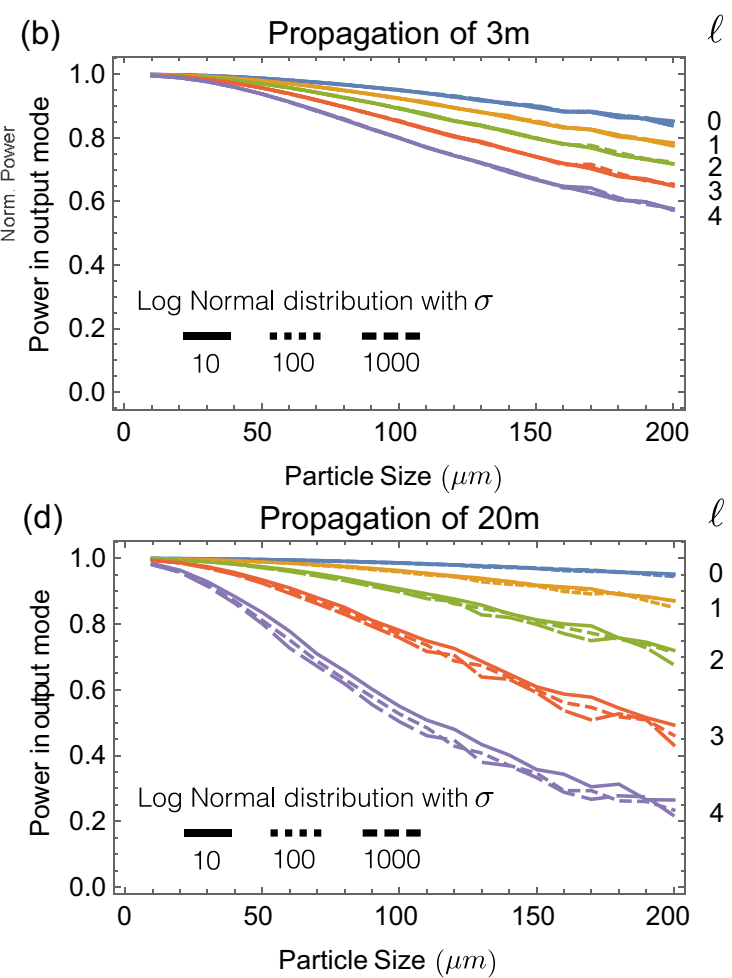

FIG. 6. (a) Considering the range of input OAM modes with values between $\ell=0$ and $\ell=4$, a matrix can be determined to indicate the distribution of power in the output modes. Using these matrices, one can determine the power transfer for a range of particulate sizes by calculating the eigenvalues of a 2D matrix for each simulated value. We simulate the scattering of OAM modes for average particulate sizes over the range 10 to $200 \mu \mathrm{m}$ and determine the eigenvalues corresponding to $\ell=0$ through $\ell=4$ for (b) $3 \mathrm{~m}$, (c) $12 \mathrm{~m}$, and (d) $20 \mathrm{~m}$. For each average particle size we consider a random variance with a log-normal distribution, Eq. (9), with $\sigma=10,1000$, and 10000 . Each point is determined by averaging 1000 random scattering screens.

not expect considerable modal deformation. The simulated channel was $3 \mathrm{~m}$ in length with 30 consecutive absorbing screens. Uniform distributions of particles with diameters of 11 and $50 \mu \mathrm{m}$ were modeled, resulting in a total number of distributed particles of $4.13 \times 10^{5}$ and 4140 , respectively (Fig. 5). In each case the total channel attenuation was $P_{\text {loss }}=$ $20 \mathrm{~dB}$, and the power spectrum was the average of 1000 instances of randomly distributed particles. The results show a distinct increase in cross talk as particle size is increased.

To characterize the distortion of the optical wave front, we define the power transfer matrix for a particular OAM mode with value $\ell_{\text {in }}$ that will have their power distributed into various output modes $\ell_{\text {out }}$. This matrix has the form

$$
P_{\ell_{\text {in }}, \ell_{\text {out }}}=\left(\begin{array}{cccc}
P_{4,0} & P_{4,1} & \cdots & P_{4,4} \\
P_{3,0} & P_{3,1} & \cdots & P_{3,4} \\
\vdots & \vdots & \ddots & \vdots \\
P_{0,0} & P_{0,0} & \cdots & P_{0,4}
\end{array}\right) .
$$

To determine the optical degradation, i.e., optical power that is transferred to the off-diagonal components, we calculate the eigenvalues of this matrix, which we name $P_{C T}(\ell)$. For particles with diameters of 11 and $50 \mu \mathrm{m}$ and input modes with $\ell=2$ and $p=0$ and $\ell=4$ and $p=0$, the results are shown in Fig. 5. It should be noted that for many communication applications this level of cross talk would be negligible and would allow for a space-division multiplexing in that channel for $11-\mu \mathrm{m}$ particles for both modes; however, for $50-\mu \mathrm{m}$ particles considerable cross talk is measured.

For most systems, the operational length would be expected to be considerably longer than $3 \mathrm{~m}$. To explore the connection between channel length and measured cross talk from distributed particles, we consider a channel with a fixed total attenuation of $A_{T}=20 \mathrm{~dB}$, the same as for the $3-\mathrm{m}$ underwater link. We investigate propagation lengths of 3,12 , and $20 \mathrm{~m}$ (Fig. 6). We consider LG modes with a range of $\ell$ values and particle sizes to assess any OAM-dependent change in performance. It can be seen in Fig. 6 that increasing both distance and $\ell$ dramatically increases $P_{C T}$. These results indicate that OAM mode cross talk has a strong dependence on particle size and increased cross talk at longer link lengths. In any real-world environment scattering-channel particulates will have a random distribution in size. To simulate the relatively narrow size distributions used in our experiments, we consider a log-normal distribution with the form

$$
R(n)=\frac{1}{\sigma \sqrt{2 \pi} x} \exp \left(\frac{-\left(\ln n-r_{p}\right)^{2}}{2 \sigma^{2}}\right),
$$

where $n$ varies from zero to $N_{p}$ in steps of 1 and $\sigma$ is the standard deviation of the distribution.

The physical reason for the presented optical behavior is that the propagation distance after a small perturbation is critical in determining the resulting phase-front distortion. Each 
absorbing particle creates diffraction at its edges, generating additional spatial modes in the optical field from deflection of the wave front similar in form to an Airy disk [27]. During propagation these phase fluctuations will diverge at a rate different from the input mode, resulting in an increased modal degradation that increases $P_{C T}$. Successive interactions with absorbing particles over long distances or greater in diameter will result in compounded diffractive effects, as seen in Fig. 6. These results highlight that experimental and numerical simulations of interaction of particles with structured beams must be completed over distance scales similar to end-use cases in order to be valid. Further, these results present an exciting opportunity to use OAM modes as indicators for particulate size, as monitoring the difference in measured cross talk for a set of OAM modes at known distances directly indicates the particle size. This could be widely used as a long-distance particulate sensor. For large particles, variations in $P_{C T}$ are easily observable; however, for smaller particles similar to those that occur naturally in air or water bodies with a size scale of $2 \mu \mathrm{m}$ or less, this would require efficient sorting techniques that can detect accurately variations in $P_{C T} \leqslant 1 \times$ $10^{-3}$. Many environmental situations will have a broad range of particle sizes, which would potentially mean aberrations from larger particles could limit the visibility of smaller particles. However, this is an interesting area of future study.

\section{CONCLUSION}

We studied the propagation dynamics of LG modes propagating in a submersed optical channel that simulates the conditions of coastal water containing a high density of silt particles. An optical modeling technique was developed to simulate the propagation of optical beams through a scattering environment, using a cascaded absorbing-screen approach combined with a plane-wave decomposition beam propagation method. The modeling was initially compared with results from a short submersed optical channel similar to that widely used for system testing, confirming the model's validity. Building on this, we utilized the model to extend the propagation distance and vary the particulate size. We showed that the OAM mode is largely unperturbed at short distances. However, at long distances small particulates result in a distinctive cross-talk profile that is influenced by the diameter of randomly distributed particles in the optical channel. These results indicate a mode-dependant interaction for beams that carry OAM and open the possibility to use structured beams as a probe for scattering media and suggest that they could be a useful tool for solving complex inversion problems such as particle sizing in scattering environments in future studies.

\section{ACKNOWLEDGMENTS}

The authors would like to thank E. Johnson and L. Uranga for useful discussions. M.P.J.L. would like to acknowledge the Royal Academy of Engineering, EPSRC, and the Scottish University Physics Alliance for their support. The work was supported by the Royal Academy of Engineering, EPSRC Award No. EP/N032853/1, and NERC Award No. $\mathrm{NE} / \mathrm{P} 003265 / 1$, and this project has received funding from the European Union's Horizon 2020 research and innovation program under the Future and Emerging Technologies Open grant agreement SuperPixels No. 829116. A.M.Y. thanks the Leverhulme Trust for the award of Leverhulme Trust Research Project Grant No. RPG-2017-048.
[1] D. J. Richardson, J. M. Fini, and L. E. Nelson, Space-division multiplexing in optical fibres, Nat. Photonics 7, 354 (2013).

[2] A. E. Willner, H. Huang, Y. Yan, Y. Ren, N. Ahmed, G. Xie, C. Bao, L. Li, Y. Cao, Z. Zhao, J. Wang, M. P. J. Lavery, M. Tur, S. Ramachandran, A. F. Molisch, N. Ashrafi, and S. Ashrafi, Optical communications using orbital angular momentum beams, Adv. Opt. Photonics 7, 66 (2015).

[3] N. Bozinovic, Y. Yue, Y. Ren, M. Tur, P. Kristensen, H. Huang, A. E. Willner, and S. Ramachandran, Terabit-scale orbital angular momentum mode division multiplexing in fibers, Science 340, 1545 (2013).

[4] S. Viola, M. Valyrakis, A. E. Kelly, and M. P. J. Lavery, Submersed free-space propagation of beams carrying orbital angular momentum, Proc. SPIE 9991, 999103 (2016).

[5] S. Watson, M. Tan, S. P. Najda, P. Perlin, M. Leszczynski, G. Targowski, S. Grzanka, and A. E. Kelly, Visible light communications using a directly modulated $422 \mathrm{~nm} \mathrm{GaN}$ laser diode, Opt. Lett. 38, 3792 (2013).

[6] F. Hanson and S. Radic, High bandwidth underwater optical communication, Appl. Opt. 47, 277 (2008).

[7] Y. Ren, L. Li, Z. Wang, S. M. Kamali, E. Arbabi, A. Arbabi, Z. Zhao, G. Xie, Y. Cao, N. Ahmed, Y. Yan, C. Liu, A. J. Willner, S. Ashrafi, M. Tur, A. Faraon, and A. E. Willner, Orbital angular momentum-based space division multiplexing for high-capacity underwater optical communications, Sci. Rep. 6, 33306 (2016).

[8] A. M. Yao and M. J. Padgett, Orbital angular momentum: Origins, behavior and applications, Adv. Opt. Photonics 3, 161 (2011).

[9] G. Gibson, J. Courtial, M. J. Padgett, M. V. Vasnetsov, V. A. Pas'ko, S. M. Barnett, and S. Franke-Arnold, Free-space information transfer using light beams carrying orbital angular momentum, Opt. Express 12, 5448 (2004).

[10] H. Huang, G. Xie, Y. Yan, N. Ahmed, Y. Ren, Y. Yue, D. Rogawski, M. J. Willner, B. I. Erkmen, K. M. Birnbaum, S. J. Dolinar, M. P. J. Lavery, M. J. Padgett, M. Tur, and A. E. Willner, $100 \mathrm{Tbit} / \mathrm{s}$ free-space data link enabled by three-dimensional multiplexing of orbital angular momentum, polarization, and wavelength, Opt. Lett. 39, 197 (2014).

[11] M. P. J. Lavery, H. Huang, Y. Ren, G. Xie, and A. E. Willner, Demonstration of a $280 \mathrm{Gbit} / \mathrm{s}$ free-space space-divisionmultiplexing communications link utilizing plane-wave spatial multiplexing, Opt. Lett. 41, 851 (2016).

[12] M. P. J. Lavery, M. M. Abadi, R. Bauer, G. Brambilla, L. Cheng, M. A. Cox, A. Dudley, A. D. Ellis, N. K. Fontaine, A. E. Kelly, C. Marquardt, S. Matlhane, B. Ndagano, F. Petruccione, R. Slavík, F. Romanato, C. Rosales-Guzmán, F. S. Roux, K. Roux, 
J. Wang, and A. Forbes, Tackling Africa's digital divide, Nat. Photonics 12, 249 (2018).

[13] M. P. J. Lavery, C. Peuntinger, K. Günthner, P. Banzer, D. Elser, R. W. Boyd, M. J. Padgett, C. Marquardt, and G. Leuchs, Freespace propagation of high-dimensional structured optical fields in an urban environment, Sci. Adv. 3, e1700552 (2017).

[14] O. Korotkova, Light propagation in a turbulent ocean, Prog. Opt. 64, 1 (2019).

[15] M. P. J. Lavery, Vortex instability in turbulent free-space propagation, New J. Phys. 20, 043023 (2018).

[16] V. I. Tatarski, Wave Propagation in a Turbulent Medium (McGraw-Hill, New York, 1961).

[17] D. L. Fried, Statistics of a geometric representation of wavefront distortion, J. Opt. Soc. Am. 55, 1427 (1965).

[18] B. Rodenburg, M. P. J. Lavery, M. Malik, M. N. O’Sullivan, M. Mirhosseini, D. J. Robertson, M. J. Padgett, and R. W. Boyd, Influence of atmospheric turbulence on states of light carrying orbital angular momentum, Opt. Lett. 37, 3735 (2012).

[19] G. A. Tyler and R. W. Boyd, Influence of atmospheric turbulence on the propagation of quantum states of light carrying orbital angular momentum, Opt. Lett. 34, 142 (2009).

[20] N. R. Heckenberg, R. McDuff, C. P. Smith, and A. G. White, Generation of optical-phase singularities by computergenerated holograms, Opt. Lett. 17, 221 (1992).
[21] G. C. G. Berkhout, M. P. J. Lavery, J. Courtial, M. W. Beijersbergen, and M. J. Padgett, Efficient Sorting of Orbital Angular Momentum States of Light, Phys. Rev. Lett. 105, 153601 (2010).

[22] M. P. J. Lavery, D. J. Robertson, G. C. G. Berkhout, G. D. Love, M. J. Padgett, and J. Courtial, Refractive elements for the measurement of the orbital angular momentum of a single photon, Opt. Express 20, 2110 (2012).

[23] M. P. J. Lavery, G. C. G. Berkhout, J. Courtial, and M. J. Padgett, Measurement of the light orbital angular momentum spectrum using an optical geometric transformation, J. Opt. 13, 064006 (2011).

[24] J. G. Ayala-Landeros, V. Saucedo-Rivalcoba S. BribiescaVasquez, V. M. Castaño, A. L. Martínez-Hernández, and C. Velasco-Santos, Influence of corn flour as pore forming agent on porous ceramic material based mullite: Morphology and mechanical properties, Sci. Sintering 48, 29 (2016).

[25] M. J. Padgett, The Poynting vector in Laguerre-Gaussian laser modes, Opt. Commun. 121, 36 (1995).

[26] L. Allen, M. W. Beijersbergen, R. J. C. Spreeuw, and J. P. Woerdman, Orbital angular-momentum of light and the transformation of Laguerre-Gaussian laser modes, Phys. Rev. A 45, 8185 (1992).

[27] M. Born and E. Wolf, Principles of Optics (Cambridge University Press, Cambridge, 1999). 